Research Article

\title{
Drug utilization study of atopic dermatitis in a tertiary care hospital
}

\author{
Veena Rani Vemuri ${ }^{1 *}$, Usha V. Nayak ${ }^{2}$
}

${ }^{1}$ Department of Pharmacology, Terna Medical College, Nerul, Navi Mumbai, India

${ }^{2}$ Department of Pharmacology, KJ Somaiya Medical College, Chunnabhati, Near Everard Nagar, Sion East, Mumbai, India

\begin{abstract}
Background: Atopic dermatitis is a chronic relapsing hypersensitivity manifestation of the skin which has shown an increasing prevalence world over. It ranges from a mild atopic itch to an unrelenting chronic eczema. Its prevalence which is on the rise necessitates a rational approach to the diagnosis and treatment.

Methods: A prospective study was conducted for a period of six months and 245 prescriptions were collected after obtaining requisite permission and a proper informed consent. The data was analysed by using WHO drug use indicators.

Results: Of the 245 prescriptions analysed, there was a female predominance with a female to male ratio of $1.63: 1$. Age group presenting more with the symptoms of atopic dermatitis was 20 to 29 years. Antihistamines were the most commonly prescribed medication (221), Emollients were present in 193 prescriptions. Topical steroids were received by 150 patients of whom 51 were prescribed as an FDC. Tacrolimus was given to 17 patients.

Conclusions: It is essential to rule out helminthic infestation, scabies and seborrhoea dermatitis to make a proper diagnosis of atopic dermatitis. More generic prescribing wherever possible might help to reduce the cost per patient.
\end{abstract}

Received: 07 July 2016

Accepted: 06 August 2016

*Correspondence to:

Dr. Veena Rani Vemuri,

Email: uv_s2@yahoo.co.in

Copyright: (C) the author(s), publisher and licensee Medip Academy. This is an openaccess article distributed under the terms of the Creative Commons Attribution NonCommercial License, which permits unrestricted noncommercial use, distribution, and reproduction in any medium, provided the original work is properly cited.

Keywords: AD, Atopic eczema, Drug utilization study, Topical corticosteroids, Topical calcineurin inhibitors, Tacrolimus

\section{INTRODUCTION}

Atopic dermatitis is a chronic or chronically relapsing hypersensitive manifestation of the skin with itching as a predominant feature. A gradual increase in the prevalence of atopic dermatitis has been observed in recent times. It can be ascribed to environmental changes consequent to rapid development all over the world. A rising trend in Atopic Dermatitis has been observed in India also in last four decades. ${ }^{1}$ Topical Corticosteroids form a major part of allergic skin disorder treatment. But with the use of topical calcineurin inhibitors there is a decreased use of topical corticosteroids.

Drug utilization study is an essential part of pharmacoepidemiology as it describes the extent, nature and determinants of drug exposure. It helps in providing a hypothesis to determine whether there is an underuse or overuse of drugs and whether pedagogic interventions (education) are required. Rational use of medications helps reduce the adverse effects.

This study is aimed at analysing the prescriptions of patients presenting with atopic dermatitis and Eczema at a dermatological OPD of a tertiary care hospital.

\section{METHODS}

A prospective study was carried out in the outpatient dermatology department of a tertiary care hospital in Navi Mumbai after obtaining requisite permission from the Institutional Ethics Committee. Confidentiality and anonymity of the patients was maintained. A proper informed consent was taken after explaining the study to the patients in a language they could understand.

A total of 245 prescriptions issued to patients attending the dermatology outpatient department were entered in 
the case record forms following the consultation. The prescriptions were collected for a period of six months.

\section{Prescriptions were analysed using following indicators}

- The prescriptions were tabulated according to the age group of the patients

- Average number of drugs per encounter

- Percentage of fixed dose combinations was calculated and compared with the number of single preparations. The rationality and purpose was analyzed

- The drugs were classified into different groups and analysis of the more common drug groups was done

- Drugs were further classified according to the route of administration

- Total number of prescriptions containing FDCs with corticosteroids was calculated.

Table 1: Table of results.

\begin{tabular}{|ll|}
\hline \multicolumn{2}{|c|}{ Table of results } \\
\hline Total number of prescriptions & 245 \\
\hline Total number of drugs prescribed & 703 \\
\hline Average number of drugs per prescription & 2.87 \\
\hline Ratio of females to males & $1.63: 1$ \\
\hline Oral formulations & 295 \\
\hline Topical preparations & 357 \\
\hline Fixed dose combinations & 51 \\
\hline
\end{tabular}

Table 2: Age wise distribution of study sample.

\begin{tabular}{|ll|}
\hline Age wise distribution & Number of patients \\
\hline$<9$ years & 27 \\
\hline 10 to 19 & 8 \\
\hline 20 to 29 & 63 \\
\hline 30 to 39 & 52 \\
\hline 40 to 49 & 40 \\
\hline 50 to 59 & 15 \\
\hline 60 and above & 40 \\
\hline Total & $\mathbf{2 4 5}$ \\
\hline
\end{tabular}

\section{RESULTS}

Of total 245 cases, 151(62\%) were females and 94 males $(38 \%)$. There were $63(25.71 \%)$ in the age group of $20-29$ years showing a larger prevalence in this age group. Second largest group is between 30-39 years with 52 cases $(21.22 \%)$.

In 245 prescriptions, 703 drugs were prescribed. Average number of drugs per prescription in our study was found to be 2.87 . All drugs were prescribed by brand names.
Table 3: Number of prescriptions with different drug classes.

\begin{tabular}{|ll|}
\hline Drug classes & Number of prescriptions \\
\hline Antihistamines & 221 \\
\hline Oral antibiotics & 18 \\
\hline Oral antifungal & 17 \\
\hline Vitamin supplements & 18 \\
\hline Topical antibiotics & 17 \\
\hline Topical antifungal & 31 \\
\hline Topical steroids & 99 \\
\hline Emmolients & 193 \\
\hline FDC with steroid & 51 \\
\hline Tacrolimus & 17 \\
\hline Total & $\mathbf{7 0 3}$ \\
\hline
\end{tabular}

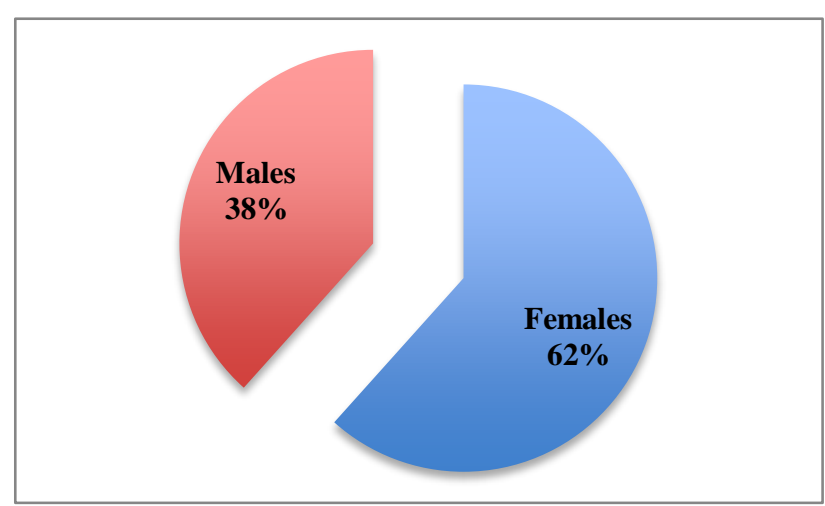

Figure 1: Gender wise distribution of study population.

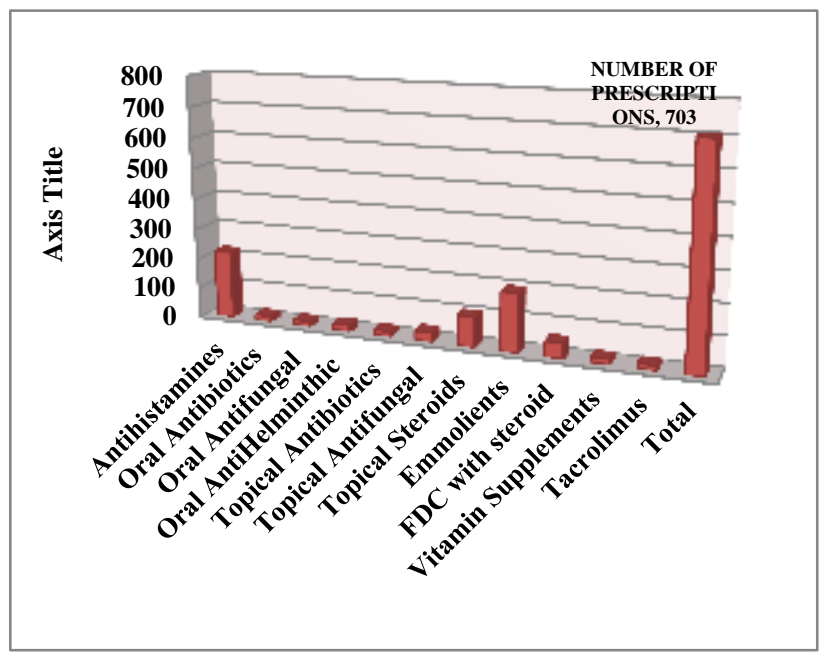

Figure 2: Frequency of prescription of drugs from various groups.

Largest group of drugs prescribed were Antihistamines (221). They constituted $31.4 \%$ of the drugs prescribed. Most commonly prescribed antihistamine was levocetirizine. But the drug of choice in the paediatric age group was Hydroxyzine as a syrup formulation. Polypharmacy was more common while prescribing these drugs. 
Emollients were prescribed to 193 patients. They are the main stay of maintenance therapy and therefore justified.

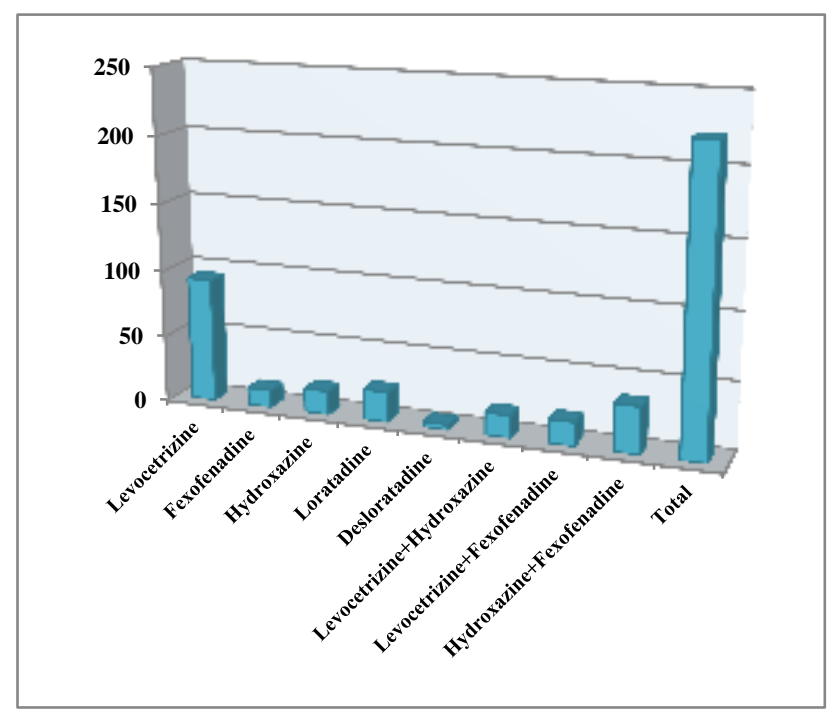

Figure 3: Frequency of prescription of different antihistamines.

Table 4: Frequency of prescription of various antihistamines.

\begin{tabular}{|ll|}
\hline Antihistamines & $\begin{array}{l}\text { Number of } \\
\text { prescriptions }\end{array}$ \\
\hline Levocetrizine & 93 \\
\hline Fexofenadine & 13 \\
\hline Hydroxazine & 18 \\
\hline Loratadine & 23 \\
\hline Desloratadine & 4 \\
\hline Levocetrizine+Hydroxazine & 17 \\
\hline Levocetrizine+Fexofenadine & 18 \\
\hline Hydroxazine+Fexofenadine & 35 \\
\hline Total & $\mathbf{2 2 1}$ \\
\hline
\end{tabular}

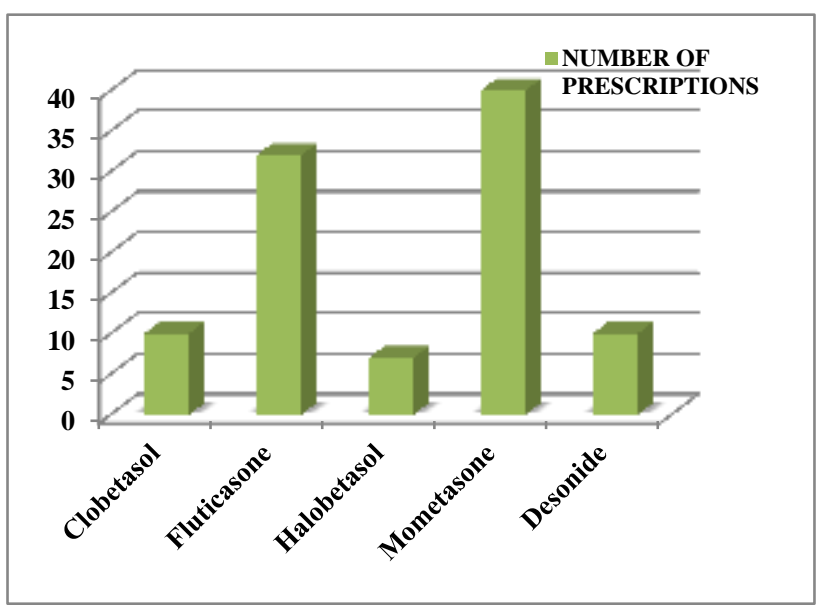

Figure 4: Frequency of prescription of various topical corticosteroids.
Oral Antibiotic was received by 18 patients. Only two were drugs of choice, azithromycin and cefadroxil. 17 patients received topical antibiotics. Fusidic acid and clindamycin were the two topical antibiotics prescribed. 31 patients received a topical antifungal and 17 patients received an oral antifungal agent.

Oral antihelminthic was received by 21 patients, and tacrolimus by 17 patients.

150 patients received Topical Corticosteroids, of which 99 were single preparations and 51 were as an FDC. Mometasone Furoate was prescribed to 40 patients and clobetasol which is a potent topical corticosteroid was given to 10 patients.

\section{DISCUSSION}

There has been a constant increase in the number of cases presenting with atopic dermatitis. It often begins in early childhood and has a waxing and waning course. There is usually an associated family history of atopy. ${ }^{4}$

Atopic dermatitis was the commonest dermatosis in children registered to a paediatric dermatology clinic where it constituted $29.9 \%$ of all registered patients. ${ }^{5}$

Gender ratio has varied greatly between the studies though many have reported a male predominance $2.25: 1$ for infants. ${ }^{5,7}$ In our study we found that there was a female predominance with a female to male ratio of 1.63:1.

Average number of drugs per prescription is an important index of prescription audit. An average of 2.87 of our study was in confirmation with some of the other hospital studies done in India which showed 2-3 drugs per prescription. $^{1-3,5,6}$

Atopic dermatitis encompasses an array of features. They can be broadly classified into: atopic itch, the atopic dry skin, the atopic eczema and the stigmata of atopic dermatitis. Treatment of atopic dermatitis includes emollient therapy, corticosteroids, topical calcineurin inhibitors and antipruritic therapy with antihistamines. Effective topical therapy depends on three fundamental principles: strength, dosage and correct application.

Topical glucocorticoids are a first-line anti-inflammatory treatment, according to the needs of the patient (pruritus, sleeplessness, new flare). In our study 150 patients received topical steroids, of which 99 were single preparations and 51 were as an FDC. Mometasone furoate which is of medium potency was the most preferred topical steroid. Clobetasol which is a potent corticosteroid was given to 10 patients. Desonide was the preferred drug for children. Low potency agents are preferred in infants also. 
Topical calcineurin Inhibitors, tacrolimus ointment and pimecrolimus cream, are licenced for topical eczema treatment. In our study 17 patients received tacrolimus. A double blind, multi centric, vehicle controlled trial conducted in children from 7 to 16 years age concluded that tacrolimus was safe in children with Atopic dermatitis. ${ }^{11}$ A meta-analysis of randomized controlled trials comparing tacrolimus and pimecrolimus was published in 2005. Tacrolimus was found to be superior in efficacy when compared to placebo. But since long term safety studies were absent there was no conclusive proof that they were superior to topical glucocorticoids. ${ }^{8}$ A randomized double blind multi centric trial that compared three concentrations of tacrolimus ointment - $0.03 \%$, $0.1 \%$ and $0.3 \%$ found that it was effective in controlling the symptoms of Atopic dermatitis with only burning sensation as a side effect. ${ }^{12}$ A study comparing tacrolimus $0.03 \%$ and $0.1 \%$ ointment to $0.1 \%$ of Hydrocortisone Butyrate in adult patients found that they were both similar in efficacy. ${ }^{13}$

It is the most important clinical symptom in atopic Dermatitis, which has an emotional impact on the patient. Antihistamines have been used in an attempt to relieve pruritus in patients with atopic dermatitis. However a meta-analysis conducted in 1999 had concluded that there is no evidence to support the relief of symptom of itch in these patients. They had also mentioned that the sedating Antihistamines were found to be useful in some studies because of their capacity to induce drowsiness or sedation. $^{14}$

A research into the presence and impact of Atopic dermatitis associated staphylococcal biofilm as a cause of occlusion of sweat glands leading to inflammation and pruritus was conducted in pennsylvania. It concludes that staphlococci were present in $93 \%$ of samples from atopic dermatitis lesions. ${ }^{15}$ There is evidence to suggest that staphylococci can impact diseases severity. ${ }^{16}$ In severe exacerbations systemic antibiotic treatment may be helpful.

Prescribing an oral anti-helminthic for a patient especially in children may be justified as an infestation can present with an itch. Prescribing an Antifungal depends on the clinician's discretion.

All prescriptions recorded the route of administration, dose, and frequency of administration and duration of treatment. This positive observation would be a sign of good prescribing patterns in this outpatient department.

Limitation of this study was a one-time access and analysis of prescriptions. A more detailed study may be helpful in knowing seasonal variations, prevalence in infants and children and improvement of symptoms with treatment. Quality of life measurements may help in analysing the disease impact on daily life of the patients.

\section{CONCLUSION}

It is essential to rule out helminthic infestation, scabies and seborrheic dermatitis to make a proper diagnosis of atopic dermatitis. More generic prescribing wherever possible might help to reduce the cost per patient.

Funding: No funding sources

Conflict of interest: None declared

Ethical approval: The study was approved by the Institutional Ethics Committee

\section{REFERENCES}

1. Dhar S. Atopic dermatitis: Indian scenario. Indian J Dermatol Venereol Leprol. 1999;65:253-7.

2. Sharma P, Kapoor B. Study of prescribing pattern for rational drug therapy. JK Science. 2003;5(3):107-9.

3. Tikoo D, Chopra SC, Kaushal S, Dogra A. Evaluation of drug use pattern in dermatology as a tool to promote rational prescribing. JK science. 2011;13(3):128-31.

4. Lebwohl MG, Del Rosso JQ, Abramovits W, Berman B, Cohen DE, Guttman-Yassky E, et al. Pathways to managing atopic dermatitis: consensus from the experts. J Clin Aesthet Dermatol. 2013;6(7):S2-S18.

5. Rashmi S, Amrinder K. J Clinico-Epidemiological Profile And Factors Affecting Severity Of Atopic Dermatitis In North Indian Children. 2004;49(3):11722.

6. Dhar S, Kanwar AJ. Epidemiology and clinical pattern of atopic dermatitis in North Indian pediatric population. Pediatr Dermatol. 1998;15:347-51.

7. Dhar S, Mandal B, Ghosh A. Epidemiology and clinical pattern of atopic dermatitis in 100 children seen in city hospital. Indian J Dermatol. 2002;47:2024.

8. Karthikeyan K, Thappa DM, Jeevankumar B. Pattern of pediatric dermatoses in a referral center in South India. Indian Pediatr. 2004;41:373-7.

9. Ashcroft Darren M, Paul D, Ruth G, Ken S, Williams Hywel C. Efficacy and tolerability of topical pimecrolimus and tacrolimus in the treatment of atopic dermatitis: meta-analysis of randomised controlled trials. BMJ. 2005;330:516.

10. Klein PA, Clark RA. An evidence-based review of the efficacy of antihistamines in relieving pruritus in atopic dermatitis. Arch Dermatol. 1999;135:1522-5.

11. Boguniewicz M, Fiedler VC, Raimer S, Lawrence I, Leung D, Hanifin J. A randomized, vehiclecontrolled trial of tacrolimus ointment for treatment of atopic dermatitis in children. J Allergy Clin Immunol. 1998;102:637-44.

12. Ruzicka T, Bieber T, Schopf E, Rubins A, Dobozy A, Bos JD, et al. A short-term trial of tacrolimus ointment for atopic dermatitis. European Tacrolimus Multicenter Atopic Dermatitis Study Group. N Engl J Med. 1997;337:816-21.

13. Reitamo S, Rustin M, Ruzicka T, Cambazard F, Kalimo K, Friedmann PS, et al. Efficacy and safety 
of tacrolimus ointment compared with that of hydrocortisone butyrate ointment in adult patients with atopic dermatitis. J Allergy Clin Immunol. 2002;109:547-55.

14. Klein PA, Richard AF, Clark. An evidence based review of efficacy of antihistamines in relieving pruritus in Atopic dermatitis. Arch Dermatol. 1999;135:1522-5.
15. Allen HB, Vaze ND, Choi C, Hailu T, Tulbert BH, Cusack CA, et al. The presence and impact of biofilm-producing staphylococci in atopic dermatitis JAMA dermatol. 2014;150(3):260-5.

16. Bunikowski R, Mielke M, Skarabis H, Worm M, Anagnostopoulos I, Kolde G, et al. Evidence for a disease promoting effect of Staphylococcus aureusderived exotoxins in atopic dermatitis. J Allergy Clin Immunol. 2000;105:814-9.

Cite this article as: Vemuri VR, Nayak UV. Drug utilization study of atopic dermatitis in a tertiary care hospital. Int J Basic Clin Pharmacol 2016;5:2061-5. 